\title{
Therapeutic strategies for allergic diseases
}

\author{
Peter J. Barnes
}

Many drugs are now in development for the treatment of atopic diseases, including asthma, allergic rhinitis and atopic dermatitis. These treatments are based on improvements in existing therapies or on a better understanding of the cellular and molecular mechanisms involved in atopic diseases. Although most attention has been focused on asthma, treatments that inhibit the atopic disease process would have application to all atopic diseases, as they often coincide. Most of the many new therapies in development are aimed at inhibiting components of the allergic inflammatory response, but in the future there are real possibilities for the development of preventative and even curative treatments.

Atopic diseases ${ }^{\star}$ account for a large proportion of health care spending in industrialized countries, as these conditions are common, persistent and currently incurable. There has been an enormous investment by the pharmaceutical companies in the search for new drugs in this profitable market. Current therapies for asthma and rhinitis are effective in most patients, whereas treatment for atopic dermatitis is less effective (Table 1). Anaphylactic shock is treatable with adrenaline (epinephrine) but there is a search for preventive therapies for susceptible patients. There is a need for new treatments to deal with more severe asthma that is currently not well controlled by high doses of inhaled corticosteroids and a need for a safe oral medication that would be effective in all atopic diseases, as they often occur together. The development of safe and effective oral therapies may depend on the discovery of treatments that are more specific for the atopic disease process, in order to avoid side effects.

New therapies for allergic diseases have developed by improving existing classes of drug or by discovering new classes of drug through research. Advances in understanding the molecular mechanisms of atopy have identified several new targets that might lead to new therapies for allergic diseases in the future ${ }^{1}$.

This review summarizes some of the areas where new drugs are in development for atopic diseases. Most attention is focused on asthma, the atopic disease with the greatest clinical and economic impact, but treatments that are effective against the underlying atopic disease process might be effective against all atopic diseases, which commonly occur together.

Bronchodilators are used for symptom relief in asthma, but have no effect on the underlying inflammatory process. Inhaled $\beta_{2}$ adrenergic agonists are safe and highly effective bronchodilators and it has proved impossible to find other classes of drug that are better. They relax airway smooth muscle by increasing the concentration of cyclic AMP and by opening potassium channels. Other classes of drug that mimic these effects, such as phosphodiesterase inhibitors or potassium channel openers, have not proved to be effective as bronchodilators, as side effects have limited the dose that can be administered ${ }^{1}$. It is unlikely that novel bronchodilators that are more effective than $\beta_{2}$-agonists will be developed and all of the attention has switched to the development of treatments that suppress or prevent the atopic inflammatory process.

I begin by discussing corticosteroids, which remain by far the most effective treatment for allergic diseases, as they provide the standard against which new treatments are judged. Many new classes of drug are in development and this very diversity highlights the fact that there are many possible therapeutic targets, most of which are suppressed by corticosteroids. The simplest approach is to

* Terms in italic are defined in the glossary on p. B39. develop inhibitors of specific inflammatory mediators and, because many mediators have been implicated in atopic diseases, there are several such drugs in development. Cytokines have a critical role in the allergic inflammatory process and I review drugs that inhibit the cytokines that promote allergic inflammation, as well as cytokines that modulate the inflammatory process. I consider anti-inflammatory drugs with a broader spectrum of action, including broad-spectrum immunosuppressants, followed by drugs that may have a more selective inhibitory effect on the allergic process. Finally, I discuss treatment strategies that may prevent the development of allergic diseases.

\section{Corticosteroids}

Corticosteroids are the most effective treatment currently available for atopic diseases and high doses of oral corticosteroids would control almost every atopic patient. However, systemic side effects limit the dose that can be given over long periods, and this led to the development of topical steroids. There is little doubt that inhaled corticosteroids have revolutionized the treatment of asthma and are now first-line treatment for chronic asthma in patients of all ages and severity of disease ${ }^{2}$. Nasal steroids are also the most effective treatment for allergic rhinitis ${ }^{3}$. Topical steroids for atopic dermatitis are associated with dermal atrophy, but and this has restricted the use of corticosteroids in the management of eczema. New-generation inhaled corticosteroids for asthma, including budesonide, fluticasone propionate and mometasone furoate, have a high level of antiinflammatory action with minimal side effects, as the swallowed fraction of drug is largely removed by hepatic metabolism ${ }^{2}$. However, these drugs are absorbed from the lung or nasal mucosa and so may have some systemic effects at high doses. There has therefore been a search for corticosteroids that are metabolized locally, so that there is less risk of systemic absorption from the respiratory tract or the skin. But these 'soft steroids', such as butixocort 21-propionate and tipredane, proved to have poor efficacy in clinical studies, as they are metabolized too rapidly before they exert their antiinflammatory action. New soft steroids, such as ciclesonide, seem to be more promising ${ }^{4}$, and corticosteroids that are inactivated in plasma are now in development. Corticosteroids are highly effective in controlling atopic diseases, and there is increasing evidence that if started early they may prevent some of the irreversible airway narrowing in asthma ${ }^{2}$. However, they do not cure the disease and allergic inflammation recurs when treatment is stopped.

Advances in understanding how corticosteroids suppress inflammation at a molecular level may lead to the development of safer steroids, or drugs that mimic their key anti-inflammatory actions. Corticosteroids bind to a cytosolic glucocorticoid receptor which translocates to the nucleus and binds as a homodimer to DNA to activate genes. The principal action of corticosteroids is to 


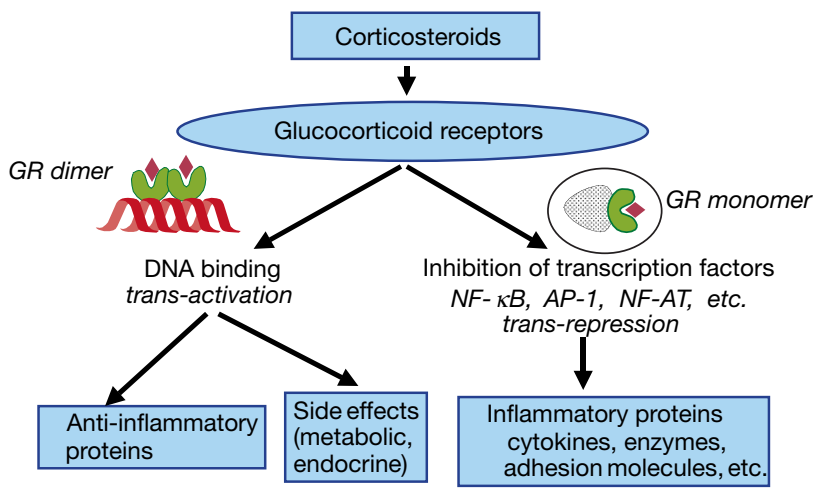

Figure 1 Dissociation of anti-inflammatory effects from side effects of corticosteroids. Corticosteroids bind to glucocorticoid receptors (GR) which dimerize to bind to DNA and increase transcription (trans-activation) and this mediates the systemic side effects of corticosteroids. Most of the anti-inflammatory effects of corticosteroids are mediated by inhibition of expression of inflammatory genes that are regulated by transcription factors,

such as activator protein-1 (AP-1), nuclear factor- $\mathrm{\kappa} B(\mathrm{NF}-\mathrm{\kappa B})$ and nuclear factor of activated T cells (NF-AT), by interaction of GR monomers with these transcription factors (trans-repression). Some corticosteroids ('dissociated steroids') are able to selectively inhibit trans-repression to a greater extent than trans-activation.

suppress multiple inflammatory genes, including cytokines, inflammatory enzymes, adhesion molecules and inflammatory mediator receptors, and this is why corticosteroids are so effective in complex inflammatory conditions. Most of the anti-inflammatory actions of corticosteroids can be accounted for by inhibiting transcription factors, such as activator protein-1 (AP-1), nuclear factor- $\kappa \mathrm{B}(\mathrm{NF}-\kappa \mathrm{B})$ and nuclear factor of activated T cells (NF-AT), that regulate inflammatory gene expression ${ }^{5}$. These effects are mediated, at least in part, by inhibition of core-histone acetylation New corticosteroids. Systemic side effects of corticosteroids are mediated largely through DNA binding, so that it may be possible to dissociate the anti-inflammatory effects, mainly mediated by transcription-factor inhibition, from their side effects (Fig. 1). Several dissociated corticosteroids have now been synthesized and a separation between trans-activation (DNA binding) and transrepression (transcription-factor inhibition) has been demonstrated in gene reporter systems and in intact cells in vitro ${ }^{6}$. Whether this will translate to in vivo differences has not yet been determined and because all corticosteroids have to bind to a single class of glucocorticoid receptor, a large separation of effects may not be possible. Identification of the major targets for corticosteroid action, such as NF- $\kappa \mathrm{B}$, activation of cAMP-response element binding protein (CREB)-binding protein (CBP) or acetylation of core histones, may be a more promising approach in the future.

\section{Mediator antagonists}

Many inflammatory mediators are involved in atopic diseases, and in asthma over 50 different mediators have been identified ${ }^{7}$. This implies that inhibitors of single mediators would be unlikely to be of major clinical benefit. Yet some mediator antagonists have been found to be useful in treating atopic diseases, indicating that these may have a more dominant role.

Antihistamines. Histamine $\mathrm{H}_{1}$-receptor antagonists have a long history in the treatment of atopic diseases. Older antihistamines such as promethazine and chlorpheniramine, which caused sedation, have now been replaced by a new generation of antihistamines such as loratadine and fexafenadine, which are much less likely to sedate. These drugs are effective in rhinitis and reduce itch in atopic dermatitis, but have no clear benefit in asthma ${ }^{8}$. New antihistamines, such as cetirizine, ebastine and astemizole, have been claimed to have additional anti-asthma effects that are not mediated through $\mathrm{H}_{1}$-receptor blockade. These effects include an inhibitory effect on eosinophil chemotaxis and adherence to endothelial cells, and inhibition of eosinophil recruitment into asthmatic airways after allergen challenge.

Antileukotrienes. Cysteinyl leukotrienes, generated from the rate- limiting enzyme 5'-lipoxygenase (5-LO), are potent bronchoconstrictors and inducers of plasma exudation, and there is some evidence that they may promote eosinophilic inflammation ${ }^{9}$. 5-LO inhibitors (zileuton) and cysteinyl-leukotriene receptor (Cys-LT 1 ) antagonists (pranlukast, zafirlukast and montelukast) have been developed for the treatment of asthma, and possibly other atopic diseases. In challenge studies they reduce allergen- and exerciseinduced asthma, as well as several other challenges. In clinical trials they improve asthma symptoms, lung function and reduce the need for rescue bronchodilator treatment ${ }^{9}$. A major advantage is that they are effective orally and, so far, there are no serious class-specific effects, although headache occurs in some patients. However, they are only weakly effective in asthma, although some patients (even with severe disease) may have a striking improvement. It is not yet possible to predict which patients will respond best, although there is some evidence that patients with aspirin-sensitive asthma do well, as may be expected form studies showing increased leukotriene production in these patients ${ }^{10}$. It is possible that genetic polymorphisms in the 5-LO pathway or Cys- $\mathrm{LT}_{1}$ receptors might predict responders in the future ${ }^{11}$. Although there are some reasons for thinking that antileukotrienes may have efficacy in allergic rhinitis, recent clinical studies have shown no benefit compared with nasal corticosteroids ${ }^{12}$.

Other mediator inhibitors. Several other inhibitors of inflammatory mediators have been tested in asthma and rhinitis with disappointing effects, including inhibitors and receptor antagonists of thromboxane synthesis, antagonists of platelet activating factor, and bradykinin and tachykinin antagonists. Inhibitors of other mediators relevant to atopic diseases are in development. There is increased production of nitric oxide (NO) in asthma and rhinitis, with evidence for increased expression of inducible NO synthase (iNOS). NO may contribute to vasodilatation and plasma exudation and has been implicated in eosinophil recruitment and survival $^{13}$. Selective inhibitors of iNOS are now in development

\begin{tabular}{|c|c|c|c|c|c|}
\hline & Asthma & $\begin{array}{l}\text { Allergic } \\
\text { rhinitis }\end{array}$ & Eczema & $\begin{array}{c}\text { Atopic } \\
\text { conjunctivitis }\end{array}$ & Anaphylaxis \\
\hline $\begin{array}{l}\text { Topical } \\
\text { corticosteroids }\end{array}$ & +++ & +++ & ++ & +++ & - \\
\hline Bronchodilators & +++ & - & - & - & $+++($ adrenaline $)$ \\
\hline Theophylline & ++ & - & - & - & - \\
\hline Cromones & + & + & - & ++ & - \\
\hline Antihistamines & - & ++ & + & - & + \\
\hline Antileukotrienes & + & - & - & - & - \\
\hline
\end{tabular}


but have not yet reached clinical studies. Endothelins have been implicated in bronchoconstriction, smooth muscle hyperplasia and fibrosis, with effects mediated by endothelin-A and endothelin-B receptors ${ }^{14}$. Mixed endothelin antagonists have now been developed although they have not yet been tested in asthma; but if their principal effect is to inhibit airway remodelling they may prove difficult to test in clinical studies.

Tryptase inhibitors. Mast-cell tryptase has several effects on airways, including increasing responsiveness of airway smooth muscle to constrictors, increasing plasma exudation, potentiating eosinophil recruitment and stimulating fibroblast proliferation. Some of these effects are mediated by activation of the proteinase-activated receptor, PAR2. A tryptase inhibitor APC366 is effective in a sheep model of allergen-induced asthma ${ }^{15}$, but was only poorly effective in asthmatic patients ${ }^{16}$. More potent tryptase inhibitors and PAR2 antagonists are now in development.

\section{Cytokine modulators}

Multiple cytokines have been implicated in the pathophysiology of atopic diseases, although some cytokines have a more critical role in atopic inflammation ${ }^{17}$. There are several possible approaches to inhibiting specific cytokines. These include the use of drugs that inhibit cytokine synthesis (glucocorticoids, cyclosporin A and tacrolimus), humanized blocking antibodies to cytokines or their receptors, soluble receptors that 'mop up' secreted cytokines to receptor antagonists and drugs that block the signal-transduction pathways activated by cytokines (Fig. 2). On the other hand, there are cytokines that suppress the allergic inflammatory process and these may have therapeutic potential.

Anti-IL-5. Interleukin (IL)-5 is crucial in orchestrating the eosinophilic inflammation of asthma ${ }^{18}$ (Fig. 3). Blocking antibodies to IL-5 inhibit eosinophilic inflammation and airway hyperresponsiveness (AHR) in animal models of asthma, including primates. This blocking effect may last for up to 3 months after a single injection, making treatment of chronic asthma with such a therapy a feasible proposition. Humanized monoclonal antibodies to IL-5 have now been developed and a single injection reduces blood eosinophils for several weeks and prevents eosinophil recruitment into the airways after allergen challenge ${ }^{19}$. However, this treatment has no effect on the early or late response to allergen challenge or on AHR, indicating that eosinophils may not be critical for these responses. Similar findings have been reported previously in mice where anti-IL-5 antibodies reduced eosinophil responses to allergen, but not AHR, whereas AHR is reduced by anti-CD4 antibody, which depletes helper $\mathrm{T}$ cells $(\mathrm{Th} \text { cells })^{20}$. Long-term clinical studies are now in progress, particularly in patients with more severe disease. Nonpeptidic IL-5-receptor antagonists would be an attractive alternative and there is a search for such compounds using molecular modelling of the IL-5-receptor $\alpha$-chain and through large-scale throughput screening.

Anti-IL-4. IL-4 is critical for the synthesis of immunoglobulin E (IgE) by B lymphocytes and is also involved in eosinophil recruitment to the airways (see review by Corry \& Kheradmand, this supplement). IL-4-receptor blocking antibodies inhibit allergeninduced AHR, goblet-cell metaplasia and pulmonary eosinophilia in a murine model $^{21}$. Inhibition of IL-4 may therefore be effective in inhibiting allergic diseases, and soluble IL-4 receptors are in clinical development as a strategy to inhibit IL-4, with some preliminary evidence of efficacy by nebulization in moderate asthma ${ }^{22}$. IL-4 and the closely related cytokine IL-13 signal through a shared surface receptor that activates a specific transcription factor, signal transducer and activator of transcription 6 (Stat-6); deletion of the Stat-6 gene has a similar effect to knock-out of the IL- 4 gene $^{23}$. This has led to a search for inhibitors of Stat-6, although it will be difficult to deliver these intracellularly. An endogenous inhibitor of STATs, suppressor of cytokine signalling (SOCS-1), is a potent inhibitor of IL-4 signalling pathways and offers a new therapeutic target ${ }^{24}$.

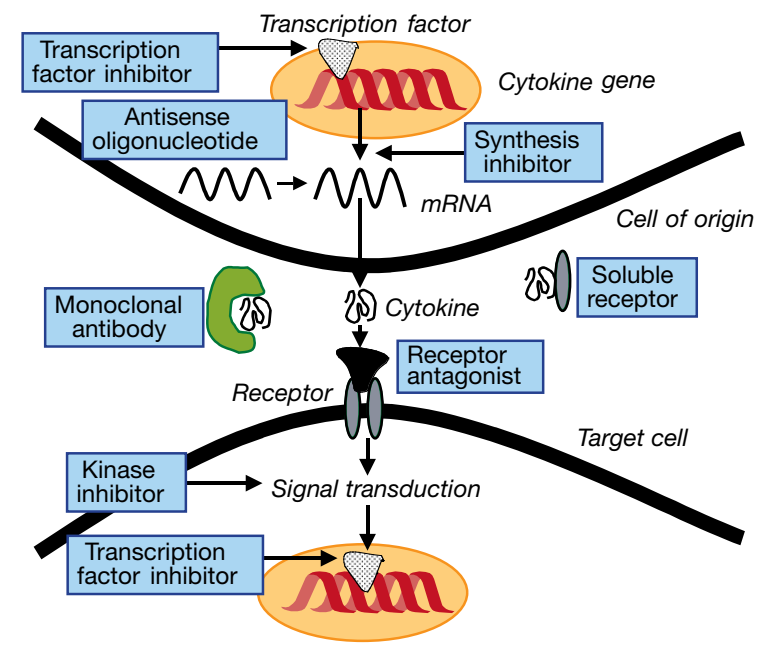

Figure 2 Strategies used to inhibit the production or effects of cytokines.

Anti-IL-13. There is increasing evidence that IL-13 in mice mimics many of the features of asthma, including AHR and mucus hypersecretion, independently of eosinophilic inflammation ${ }^{25}$. It is also a potent inducer of eotaxin secretion from airway epithelial cells $^{26}$. IL-13 signals through the IL-4-receptor $\alpha$-chain, but may also activate different intracellular pathways ${ }^{27}$, so that it may be an important target for the development of new therapies.

Anti-TNF. Tumour-necrosis factor (TNF)- $\alpha$ is expressed in asthmatic airways and may be important in amplifying asthmatic inflammation, through the activation of NF- $\mathrm{B}, \mathrm{AP}-1$ and other transcription factors. In rheumatoid arthritis and inflammatory bowel disease a blocking antibody to TNF- $\alpha$ (infliximab) has produced remarkable clinical responses, even in patients who are relatively unresponsive to steroids ${ }^{28}$. Such antibodies or soluble TNF receptors are a logical approach to asthma therapy, particularly in patients with severe disease. There is also a search for smallmolecule inhibitors of TNF, of which the most promising are inhibitors of TNF- $\alpha$ converting enzyme as these could be given orally.

Chemokine inhibitors. Chemokines are chemoattractant cytokine molecules. Chemokines such as RANTES, MCP-3, MCP-4 and eotaxin may be crucial in the recruitment of eosinophils in atopic patients; all of these chemokines act on a common receptor, the CCR3 receptor, that is expressed predominantly on eosinophils ${ }^{29}$. An antibody to human CCR3 blocks the chemotactic response of human eosinophils to all chemokines ${ }^{30}$. The modified chemokine met-RANTES similarly blocks CCR3 receptors and inhibits eosinophil chemotactic responses to chemokines ${ }^{31}$. Chemokine receptors are G-protein-coupled receptors with the typical seventransmembrane-spanning segments and are therefore have a simpler structure than the receptors for other cytokines. Non-peptide inhibitors of CCR 3 receptors are now in development and should be relatively safe as the distribution of CCR3 receptors is restricted to eosinophils, Th2 cells and basophils.

Anti-inflammatory cytokines. Some cytokines have anti-inflammatory effects in atopic inflammation and therefore have therapeutic potential $^{32}$. Although it may not be feasible or cost-effective to administer these proteins as long-term therapy, it may be possible to develop drugs that increase the release of these endogenous cytokines or activate their receptors and specific signal-transduction pathways. IL-1-receptor antagonist (IL-1ra) binds to IL-1 receptors and blocks the action of IL1 $\beta$. In experimental animals it reduces $\mathrm{AHR}^{33}$ and clinical studies are underway. 


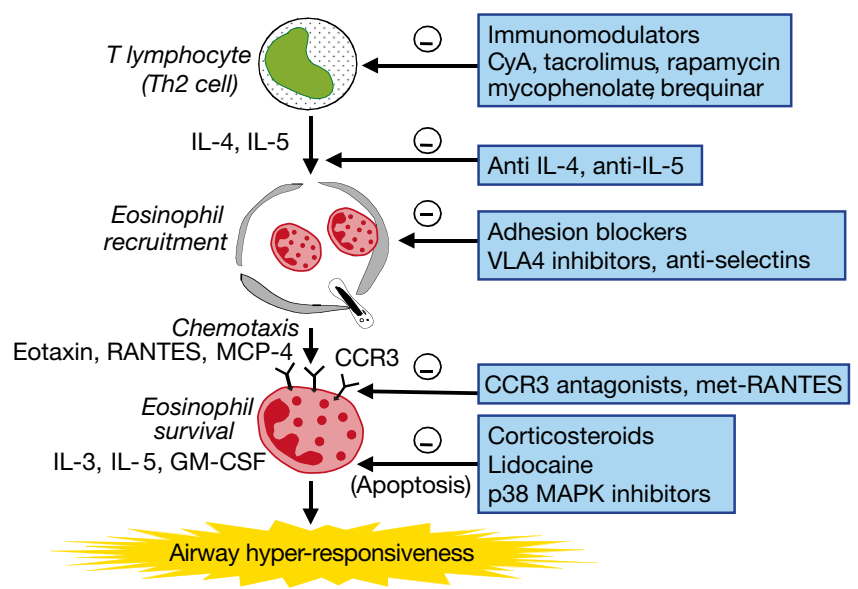

Figure 3 Inhibition of eosinophilic inflammation. Several strategies are possible to inhibit eosinophilic inflammation in tissues, including immunomodulators, inhibitors of driving cytokines (IL-4 and IL-5), inhibition of critical adhesion molecules (VLA4, selectins and
ICAM-1), blockade of chemokine receptors on eosinophils (CCR3) and induction of apoptosis.
IL-10 is a potent anti-inflammatory cytokine that inhibits the synthesis of many inflammatory proteins, including cytokines (TNF- $\alpha$, granulocyte-macrophage colony-stimulating factor (GM-CSF), IL-5 and chemokines) and inflammatory enzymes (iNOS) that are overexpressed in asthma ${ }^{34}$. Indeed, there may be a defect in IL-10 transcription and secretion from macrophages in asthma $^{35,36}$. In sensitized animals, IL-10 is effective in suppressing the inflammatory response to allergen, indicating that IL-10 might be defective in atopic diseases. Recombinant human IL-10 has proved to be effective in controlling inflammatory bowel disease, where similar cytokines are expressed, and may be given as a weekly injection $^{37}$. In the future, drugs that active the unique signaltransduction pathways activated by the IL-10 receptor or drugs that increase endogenous production of IL-10 may be developed. In mice, drugs that elevate cAMP increase IL-10 production, but this does not seem to be the case in human cells ${ }^{38}$.

Interferon- $\gamma($ IFN- $\gamma$ ) inhibits Th2 cells and should therefore reduce atopic inflammation. In sensitized animals, nebulized IFN- $\gamma$ inhibits eosinophilic inflammation induced by allergen exposure ${ }^{39}$ (see review by Corry \& Kheradmand, this supplement). But administration of IFN- $\gamma$ by nebulization to asthmatic patients did not significantly reduce eosinophilic inflammation, although this may be due to the difficulty in obtaining a high enough concentration locally in the airways ${ }^{40}$. Allergen immunotherapy increases IFN- $\gamma$ production by circulating $T$ cells in patients with clinical benefit ${ }^{41}$ and it increased the number of cells expressing IFN- $\gamma$ in nasal biopsies of patients with allergic rhinitis ${ }^{42}$.

IL-12 is the endogenous regulator of Th1-cell development and determines the balance between Th1 and Th2 cells ${ }^{43}$. IL-12 administration to rats inhibits allergen-induced inflammation ${ }^{44}$ and inhibits sensitization to allergens. IL-12 releases IFN- $\gamma$, but has additional effects on T-cell differentiation. Recombinant human IL12 has been administered to humans and has several toxic effects which are diminished by slow escalation of the dose. In mice, administration of an IL-12-allergen fusion protein results in the development of a specific Th1 response to allergens rather than the normal Th2 response with IgE formation ${ }^{45}$. This indicates the possibility of using IL-12 to provide a more specific immunotherapy, which might even be curative if applied early in the course of the atopic disease.

\section{New anti-inflammatory drugs}

There has been an intensive search for anti-inflammatory treatments that are as effective as glucocorticoids but with fewer side effects. Whereas one approach is to seek corticosteroids with a greater therapeutic effect, other approaches involve developing different classes of anti-inflammatory drugs.

Phosphodiesterase-4 inhibitors. Phosphodiesterases (PDEs) break down cyclic nucleotides that inhibit cell activation and at least nine families of enzymes have now been discovered. Theophylline, long used as an asthma treatment, is a weak but non-selective PDE inhibitor. PDE4 is the predominant family of PDEs in inflammatory cells, including mast cells, eosinophils, T lymphocytes, macrophages, and structural cells such as sensory nerves and epithelial cells $^{46}$. This has suggested that PDE4 inhibitors would be useful as an anti-inflammatory treatment in atopic disease, particularly as there is some evidence for over-expression of PDE4 in cells of atopic patients ${ }^{47}$. In animal models of asthma, PDE4 inhibitors reduce eosinophil infiltration and AHR responses to allergen ${ }^{46}$. Several PDE4 inhibitors have been tested in asthma, but with disappointing results. One PDE4 inhibitor, CDP840, had a marginal inhibitory effect on the late response to allergen, but is not being further developed $^{48}$. However, most of the PDE4 inhibitors so far tested clinically have had unacceptable side effects, particularly nausea and vomiting, and such side effects have limited the use of theophylline.

Several steps may be possible to overcome these problems. It is possible that vomiting is due to inhibition of a particular subtype of PDE4. At least four human PDE4 genes have been identified and each has several splice variants ${ }^{46,49}$. This raises the possibility that subtype-selective inhibitors may be developed that may preserve the anti-inflammatory effect, while having less propensity to side effects. PDE4D seems to be of particular importance in inflammatory cells, such as T lymphocytes and eosinophils, and may be a more specific target ${ }^{50}$; subtype-selective PDE4 inhibitors are now in development.

Transcription-factor inhibitors. Transcription factors, such as NF$\kappa \mathrm{B}$ and $\mathrm{AP}-1$, are important in the orchestration of asthmatic inflammation ${ }^{51,52}$ and this has prompted a search for specific blockers of these transcription factors. NF- $\kappa \mathrm{B}$ is inhibited naturally by the inhibitory protein $I \kappa \mathrm{B}$, which is degraded after activation by specific kinases. Inhibitors of ІкB kinases or the proteasome, the multifunctional enzyme that degrades I $\mathrm{B}$, would thus inhibit NF$\kappa \mathrm{B}$ and there is a search for such inhibitors. There are some naturally occurring inhibitors of NF- $\mathrm{kB}$, such as the fungal product gliotoxin, although this compound is toxic. There are concerns that inhibition of NF- $\kappa \mathrm{B}$ may cause side effects such as increased susceptibility to infections, which as been observed in gene-disruption studies when components of NF- $\kappa \mathrm{B}$ are inhibited ${ }^{51}$.

Cyclosporin A and tacrolimus inhibit T-lymphocyte function by blocking the transcription factor NF-AT by blocking activation of 


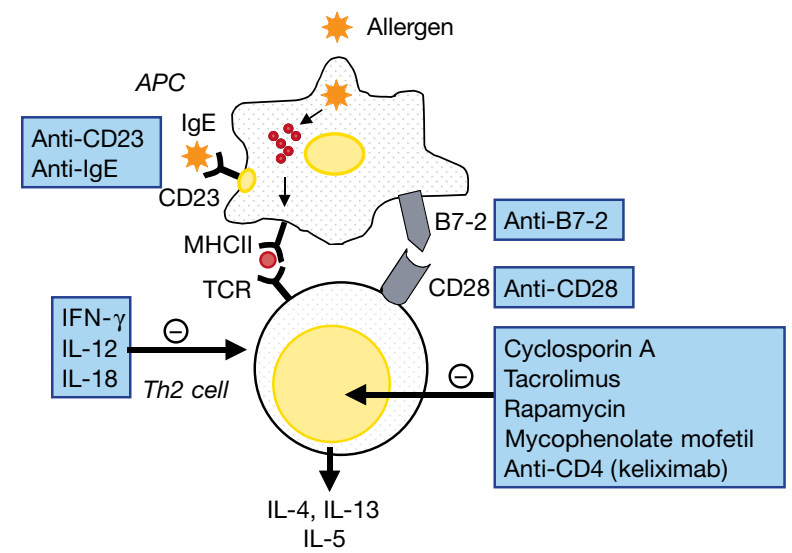

Figure 4 Inhibition of antigen-presenting cells (APCs) and Th2 lymphocytes. Therapies are based on inhibition of co-stimulatory molecules (B7-2 and CD28), inhibition of

calcineurin. This results in suppression if IL-2, IL-4, IL-5 and GMCSF and so offers therapeutic potential in atopic diseases (see below).

MAP kinase inhibitors. There are three major mitogen-activated protein (MAP) kinase pathways and there is increasing recognition that these pathways are involved in chronic inflammation ${ }^{53}$. There has been particular interest in the p38 MAP kinase pathway that is blocked by a new class of drugs, the cytokine suppressant antiinflammatory drugs (CSAIDs), such as SB203580 and RWJ67657. These drugs inhibit the synthesis of many inflammatory cytokines, chemokines and inflammatory enzymes. They appear to have a preferential inhibitory effect on synthesis of Th2 compared with Th1 cytokines, indicating their potential application in the treatment of atopic diseases ${ }^{54}$. Furthermore, p38 MAP kinase inhibitors have also been shown to decrease eosinophil survival by activating apoptotic pathways ${ }^{55}$. Whether this new class of anti-inflammatory drugs will be safe in long-term studies remains to be established.

Tyrosine kinase inhibitors. Syk kinase is a protein tyrosine kinase that has a pivotal role in signalling of the high-affinity IgE receptor (FceRI) in mast cells. In syk-deficient mice, mast-cell degranulation is inhibited, indicating that this might be an important potential target for the development of mast-cell stabilizing drugs ${ }^{56}$. Syk is also involved in antigen-receptor signalling of $\mathrm{B}$ and $\mathrm{T}$ lymphocytes and in eosinophil survival in response to IL-5 and GM-CSF ${ }^{57}$, so that $s y k$ inhibitors might have several useful beneficial effects in atopic diseases. Another tyrosine kinase lyn is upstream of $s y k$, and an inhibitor of $l y n$ kinase, PP1, has an inhibitory effect on inflammatory and mast-cell activation ${ }^{58}$. But as lyn and syk are widely distributed in the immune system, there are doubts about the longterm safety of selective inhibitors.

Immunosuppressants. T lymphocytes may be important in initiating and maintaining the inflammatory process in atopy through the release of cytokines that result in eosinophilic inflammation, indicating that T-cell inhibitors may be useful in controlling asthmatic inflammation. The non-specific immunomodulator cyclosporin A reduces the dose of oral steroids needed to control asthma in patients with severe asthma ${ }^{59}$, but its efficacy is very limited $^{60}$, and side effects, particularly nephrotoxicity, limit its clinical use. The possibility of using inhaled cyclosporin A is now being explored, as in animal studies the inhaled drug is effective in inhibiting the inflammatory response in experimental asthma ${ }^{61}$. Immunomodulators, such as tacrolimus (FK506) and rapamycin, seem to be more potent but are also toxic and may offer no real advantage. Topical tacrolimus seems to be effective in atopic dermatitis and is well tolerated ${ }^{62}$. Novel immunomodulators that inhibit purine or pyrimidine pathways, such as mycophenolate mofetil, leflunomide and brequinar sodium, may be less toxic and therefore of greater potential value in asthma therapy ${ }^{63}$.
IgE-driven APCs, and the use of non-selective immunomodulators or cytokines that tip the balance away from Th1 cells towards Th2 cells

One problem with these non-specific immunomodulators is that they inhibit both Th1 and Th2 cells, and therefore do not restore the imbalance between these cells in atopy. They also inhibit suppresser $\mathrm{T}$ cells ( $\mathrm{Tc} 1$ cells) that may modulate the inflammatory response. Selective inhibition of Th2 cells may be more effective and better tolerated and there is now a search for such drugs.

Cell adhesion blockers. Infiltration of inflammatory cells into tissues is dependent on adhesion of blood-borne inflammatory cells to endothelial cells before migration to the inflammatory site ${ }^{64}$. This depends upon specific glycoprotein adhesion molecules, including integrins and selectins, on both leukocytes and endothelial cells, which may be upregulated or show increased binding affinity in response to various inflammatory stimuli such as cytokines or lipid mediators. Monoclonal antibodies which inhibit these intracellular adhesion molecules (ICAMs) may prevent inflammatory cell infiltration. Thus a monoclonal antibody to ICAM-1 on endothelial cells prevents the eosinophil infiltration into airways and the increase in bronchial reactivity after allergen exposure in sensitized primates ${ }^{65}$, although this has not been found in other species ${ }^{66}$. The interaction between very late antigen (VLA)-4 and vascular cell-adhesion molecule (VCAM)-1 is important for eosinophil inflammation and humanized antibodies to VLA-4 $(\alpha 4 \alpha 1)$ have been developed ${ }^{67}$ (Fig. 3). Small-molecule peptide inhibitors of VLA-4 have been developed which are effective in inhibiting allergen-induced responses in sensitized sheep ${ }^{68}$. Inhibitors of selectins, particularly L-selectin, based on the structure of sialylLewis $^{\mathrm{x}}$, inhibit the influx of inflammatory cells in response to inhaled allergen in sensitized sheep ${ }^{69}$ and inhibit adhesion of human eosinophils in vitro ${ }^{70}$. Although blocking adhesion molecules is an attractive new approach to the treatment of inflammatory disease, there may be potential dangers in inhibiting immune responses leading to increased infections and increased risks of neoplasia.

\section{Specific anti-allergic drugs}

Although corticosteroids are effective in controlling atopic diseases, there are continuing concerns about systemic side effects when high doses are needed. This has prompted a search for more selective anti-inflammatory agents that would selectively target the atopic disease process.

Cromones. The cromones (sodium cromoglycate and nedocromil sodium) are the most specific anti-allergic drugs so far discovered. Topical application is effective in asthma, rhinitis and allergic conjunctivitis, but the effects are less marked that seen with topical steroids and they are effective only in mild disease. Cromones seem to have a specific action on allergic inflammation, yet their molecular mechanism of action remains obscure. Although it was believed that the primary mode of action of cromones involved inhibiting 
mast-cell mediator release, it has now been shown that they affect several other inflammatory cells and sensory nerves, including certain types of chloride channels that are expressed in mast cells $^{71}$. Sodium cromoglycate phosphorylates moesin, a specific cytoskeletal protein in mast cells, indicating a possible mechanism that may inhibit degranulation ${ }^{72}$. Both cromoglycate and nedocromil sodium must be given topically and all attempts to develop orally active drugs of this type have been unsuccessful, possibly suggesting that topical administration is critical to their efficacy.

The diuretic furosemide shares many of the actions of cromones in inhibiting indirect bronchoconstrictor challenges (such as allergen, exercise, cold air, adenosine and metabisulphite) but not direct bronchoconstriction (such as histamine and methacholine) when given by inhalation ${ }^{73}$. The mechanism of action of furosemide is not shared by the more potent loop-diuretic bumetanide, indicating that some other mechanism than the inhibition of the $\mathrm{Na}^{+} / \mathrm{K}^{+} / \mathrm{Cl}^{-}$ co-transporter must be involved. This probably involves inhibition of the same chloride channel that is inhibited by cromones. Furosemide itself does not seem to be particularly effective when given regularly by metered dose inhaler in asthma ${ }^{74}$, but it is possible that more potent and long-lasting chloride-channel blockers might be developed in the future.

Co-stimulation inhibitors. Co-stimulatory molecules may be crucial in augmenting the interaction between antigen-presenting cells (APCs) and $\mathrm{CD}^{+}{ }^{+} \mathrm{T}$ lymphocytes (see review by Corry \& Kheradmand, this supplement). The interaction between B7 and CD28 may determine whether a Th2-type cell response develops, and there is some evidence that B7-2 (CD86) skews towards a Th2 response (Fig. 4). Blocking antibodies to B7-2 inhibit the development of specific IgE, pulmonary eosinophilia and AHR in mice, whereas antibodies to B7-1 (CD80) are ineffective ${ }^{75}$. A molecule on activated T cells, CTL4, seems to act as an endogenous inhibitor of T-cell activation and CTLA4-Ig, a soluble fusion-protein construct, is also effective in blocking AHR in a murine model of asthma ${ }^{76}$. Anti-CD28, anti-B7-2 and CTLA4-Ig also block the proliferative response of $\mathrm{T}$ cells to allergen ${ }^{77}$, indicating that these are potential targets for new therapies that should be effective in all atopic diseases.

Th2-cell inhibitors. Non-selective T-cell suppressants, such as cyclosporin A and tacrolimus, may be relatively ineffective in asthma as they inhibit all types of $\mathrm{T}$ cell. $\mathrm{CD} 4^{+} \mathrm{T}$ cells have been implicated in asthma and a chimaeric antibody directed against $\mathrm{CD}^{+}$(keliximab), which reduces circulating $\mathrm{CD} 4^{+}$cells, seems to have some beneficial effect in asthma ${ }^{78}$, although long-term safety of such a treatment might be a problem. Furthermore, there is increasing evidence that $\mathrm{CD}^{+}$cells ( $\mathrm{Tc} 2$ cells), through release of IL-5 and other cytokines, might also be involved in atopic diseases, particularly in response to infections with certain viruses ${ }^{79}$. There has been a search for selective inhibitors of Th2 cells by identifying features that differentiate Th1 and Th2 cells. The transcription factor GATA-3 seems to be of particular importance in murine and human Th2 cells ${ }^{80,81}$ and may be a target for selective immunomodulatory drugs. However, an argument against strategies to control atopic disease by targeting Th2 cells is that chronic stimulation (by exposure to allergen) results in cells that are relatively resistant to immune suppression ${ }^{82}$.

Anti-IgE. Because release of mediators from mast cells in asthma is IgE-dependent (see review by Turner \& Kinet, this supplement), an attractive approach is to block the activation of IgE using blocking antibodies that do not result in cell activation. A humanized murine monoclonal antibody directed to the FceRI-binding domain of human IgE (rhuMAb-E25) reduces allergen-specific IgE after intravenous administration ${ }^{83}$. RhuMAb-E25 also reduces early and late responses to inhaled allergen and eosinophil counts in induced sputum $^{84}$. Although a reduction in early response to allergen, which is due to mast-cell activation through bound IgE, is expected, the reduction in the late response and in sputum eosinophils is unexpected, but it may be explained by blocking the effect of IgE on low-affinity IgE receptors (CD23) on APCs. Anti-IgE in mice inhibits Il-4 and IL-5 secretion and pulmonary eosinophilia by blocking Th2-cell activation in response to allergen, and this is mimicked by an anti-CD23 antibody ${ }^{85}$. Clinical studies with rhuMAb-E25 are now in progress ${ }^{86}$. Although injections of antibody may not be feasible for the long-term treatment of mild asthma, this could be a realistic therapy for patients with more severe forms of asthma or atopic dermatitis, in whom high IgE levels may be found.

\section{Preventive strategies}

Atopy seems to be due to immune deviation from Th1 to Th2 cells, which may arise because of a failure to inhibit the normal Th2 preponderance at birth, which in turn may result from environmental factors such as the Th1 response to infectious agents (see review by Holt et al., this supplement).

Specific allergen vaccination (immunotherapy). Subcutaneous injection of small amounts of purified allergen has been used form many years in the treatment of allergy. It is effective in the treatment of insect venom anaphylaxis and hay fever, and may induce prolonged remission ${ }^{87}$, but is less effective in asthma. The molecular mechanism of desensitization is unknown. Cloning of several common allergen genes has made it possible to prepare recombinant allergens for injection, although this purity may detract from their allergenicity as most natural allergens contain several proteins. Intramuscular injection of rats with plasmid DNA expressing house dust mite allergen results in its long-term expression and prevents the development of IgE responses to inhaled allergen ${ }^{88}$. This suggests that allergen gene immunization might be a useful therapeutic strategy in the future. The major allergens of peanuts, which are responsible for an increasing number of severe anaphylactic reactions, has been cloned and oral administration of microparticles of DNA complexed to chitosan in mice produces intestinal epithelial expression of the allergen and prevention of anaphylaxis after oral challenge ${ }^{89}$.

Peptide immunotherapy. Small peptide fragments of allergen (epitopes) are able to block allergen-induced T-cell responses without inducing anaphylaxis ${ }^{90}$. T-cell-derived peptides from cat allergen ( $\mathrm{fel} \mathrm{d} 1$ ) seem to be effective in blocking allergen responses to cat dander ${ }^{91}$, but may induce an isolated late response to allergen by direct T-cell activation ${ }^{92}$.

Vaccination. A relative lack of infections may be a factor that influences the development of atopy in genetically predisposed individuals. This leads to the concept that vaccination may induce protective Th1 responses to prevent sensitization and thus prevent the development of atopic diseases (see review by Holt et al., this supplement). Bacille Calmette-Guérin (BCG) vaccination has been associated with a reduction in atopic diseases in $\mathrm{Japan}^{93}$, but this has not been confirmed in a Swedish population ${ }^{94}$. BCG inoculation in mice, delivered 14 days before allergen sensitization, reduced the formation of specific IgE in response to allergen and the eosinophilic response and AHR responses to allergen, with an increase in production of IFN $-\gamma^{95}$. This has prompted several clinical trials of BCG to prevent the development of atopy. Similar results have been obtained in mice with the a single injection of heat-killed Mycobacterium vaccae, another potent inducer of Th1 responses ${ }^{96}$, and with Listeria. Lactobacillus acidophilus in yoghurt, another potential means of tipping back the balance from Th2 to Th1 cells, weakly increases IFN- $\gamma$ formation in adult asthmatic patients $^{97}$. Immunostimulatory DNA sequences, such as unmethylated cytosine-guanosine dinucleotide-containing oligonucleotides (CpG ODN), are also potent inducers of Th1 cytokines and, in mice, administration of CpG ODN increases the ratio of Th1 to Th2 cells, decreases formation of specific IgE and reduces the eosinophilic response to allergen, an effect that lasts for over 6 weeks ${ }^{98}$. These promising animal studies encourage the possibility that vaccination might prevent or cure atopic diseases in the future. 


\section{Gene therapy}

Because atopic diseases are polygenic, it is unlikely that gene therapy will be of value in long-term therapy (see review by Cookson, this supplement). However, identifying the genes involved in atopic diseases and in disease severity may identify new molecular targets and may also predict the response to different forms of therapy (pharmacogenetics). Transfer of anti-inflammatory genes may provide specific anti-inflammatory or inhibitory proteins in a convenient manner and gene transfer has been shown to be feasible in animals using viral vectors ${ }^{99}$. Anti-inflammatory proteins relevant to asthma include IL-10, IL-12 and IкB. Antisense oligonucleotides may switch off specific genes, but there are considerable problems in getting these molecules into cells. An inhaled antisense oligonucleotide directed against the adenosine $\mathrm{A}_{1}$-receptor has been shown to reduce AHR in a rabbit model of asthma, demonstrating the potential of this approach in treating asthma ${ }^{100}$. Suitable target genes may be IL- 4 or IL-5. Considering the practical problems encountered by gene therapy, this approach is unlikely in the foreseeable future, other than for proof-of-concept studies.

\section{Conclusions}

Many different therapeutic approaches to the treatment of atopic diseases may be possible, yet there have been few new drugs that have reached the clinic. Topical glucocorticoids are particularly effective as chronic treatment in atopic diseases and suppress the underlying inflammatory process. Advances in therapy would be facilitated through the development of more specific anti-allergic drugs that lack side effects. If these treatments can be taken orally this would treat asthma, rhinitis and eczema, which often coincide. The possibility of developing a 'cure' for atopy is remote, but strategies to inhibit the development of sensitization in early childhood offer such a prospect in the future.

Peter J. Barnes is in the Department of Thoracic Medicine, National Heart and Lung Institute, Imperial College, London SW3 6LY, UK (e-mail: p.j.barnes@ic.ac.uk).

1. Barnes, P. J. New drugs for asthma. Clin. Exp. Allergy 26, 738-745 (1996).

2. Barnes, P. J., Pedersen, S. \& Busse, W. W. Efficacy and safety of inhaled corticosteroids: an update. Am. J. Respir. Crit. Care Med. 157, S1-S53 (1998).

3. Mygind, N., Dahl, R., Nielsen, L. P., Hilberg, O. \& Bjerke, T. Effect of corticosteroids on nasal blockage in rhinitis measured by objective methods. Allergy 52, 39-44 (1997).

4. Taylor, D. A. et al. A dose-dependent effect of the novel inhaled corticosteroid ciclesonide on airway responsiveness to adenosine-5'-monophosphate in asthmatic patients. Am. J. Respir. Crit. Care Med. 160, 237-243 (1999).

5. Barnes, P. J. Antiinflammatory actions of glucocorticoids: molecular mechanisms. Clin. Sci. 94, 557572 (1998).

6. Vayssière, B. M. et al. Synthetic glucocorticoids that dissociate transactivation and AP-1 transrepression exhibit antiinflammatory activity in vivo. Mol. Endocrinol. 11, 1245-1255 (1997).

7. Barnes, P. J., Chung, K. F. \& Page, C. P. Inflammatory mediators of asthma: an update. Pharmacol. Rev. 50, 515-596 (1998).

8. van Ganse, E., Kaufman, L., Derde, M. P., Yernault, J. C. \& Delaunois, L. Effects of antihistamines in adult asthma: a meta-analysis of clinical trials. Eur. Respir. J. 10, 2216-2224 (1997).

9. Drazen, J. M., Israel, E. \& O'Byrne, P. M. Treatment of asthma with drugs modifying the leukotriene pathway. N. Engl. J. Med. 340, 197-206 (1999).

10. Dahlen, B. et al. Benefits from adding the 5-lipoxygenase inhibitor zileuton to conventional therapy in aspirin-intolerant asthmatics. Am. J. Respir. Crit. Care Med. 157, 1187-1194 (1998).

11. In, K. H. et al. Naturally occurring mutations in the human 5-lipoxygenase gene promoter that modify transcription factor binding and reporter gene transcription. J. Clin. Invest. 99, 1130-1137 (1997).

12. Pullerits, T., Praks, L., Skoogh, B. E., Ani, R. \& Lotvall, J. Randomized placebo-controlled study comparing a leukotriene receptor antagonist and a nasal glucocorticoid in seasonal allergic rhinitis. Am. J. Respir. Crit. Care Med. 159, 1814-1818 (1999).

13. Barnes, P. J. Nitric oxide and airway disease. Ann. Med. 27, 389-393 (1995).

14. Hay, D. W., Henry, P. J. \& Goldie, R. G. Is endothelin-1 a mediator in asthma? Am. J. Respir. Crit. Care Med. 154, 1594-1597 (1996).

15. Clark, J. M. et al. Tryptase inhibitors block allergen-induced airway and inflammatory responses in allergic sheep. Am. J. Respir. Crit. Care Med. 152, 2076-2083 (1995).

16. Krishna, M. T. et al. Effect of inhaled APC 366 on allergen-induced bronchoconstriction and airway hyperresponsivness to histamine in atopic subjects. Am. J. Respir. Crit. Care Med. 157, A456 (1998).

17. Chung, K. F. \& Barnes, P. J. Cytokines in asthma. Thorax 54, 825-857 (1999).

18. Egan, R. W., Umland, S. P., Cuss, F. M. \& Chapman, R. W. Biology of interleukin-5 and its relevance to allergic disease. Allergy 51, 71-81 (1996).

19. Leckie, M. J. et al. SB 240563, a humanized anti-IL-5 monoclonal antibody. Initial single dose safety and activity in patients with asthma. Am. J. Respir. Crit. Care Med. 159, A624 (1999).

20. Hogan, S. P. et al. A novel T cell-regulated mechanism modulating allergen-induced airways hyperreactivity in BALB/c mice independently of IL-4 and IL-5. J. Immunol. 161, 1501-1509 (1998).
21. Gavett, S. H. et al. Interleukin-4 receptor blockade prevents airway responses induced by antigen challenge in mice. Am. J. Physiol. 272, L253-L261 (1997).

22. Borish, L. C. et al. Phase I/II study of interleukin-4 receptor (IL-4R) in moderate asthma. J. Allergy Clin. Immunol. 101, S8 (1998).

23. Foster, P. S. STAT6: an intracellular target for the inhibition of allergic disease. Clin. Exp. Allergy 29, $12-16$ (1999).

24. Losman, J. A., Chen, X. P., Hilton, D. \& Rothman, P. Cutting edge: SOCS-1 is a potent inhibitor of IL-4 signal transduction. J. Immunol. 162, 3770-3774 (1999).

25. Wills-Karp, M. et al. Interleukin-13: central mediator of allergic asthma. Science 282, 2258-2261 (1998).

26. Li, L. et al. Effects of Th2 cytokines on chemokine expression in the lung: IL-13 potently induces eotaxin expression by airway epithelial cells. J. Immunol. 162, 2477-2487 (1999).

27. Chomarat, P. \& Banchereau, J. Interleukin-4 and interleukin-13: their similarities and discrepancies. Int. Rev. Immunol. 17, 1-52 (1998).

28. Feldman, M., Taylor, P., Paleolog, E., Brennan, F. M. \& Maini, R. N. Anti-TNF alpha therapy is useful in rheumatoid arthritis and Crohn's disease: analysis of the mechanism of action predicts utility in other diseases. Transplant. Proc. 30, 4126-4127 (1998).

29. Luster, A. D. Chemokines—chemotactic cytokines that mediate inflammation. N. Engl. J. Med. 338, 436-445 (1998).

30. Heath, H. et al. Chemokine receptor usage by human eosinophils. The importance of CCR3 demonstrated using an antagonistic monoclonal antibody. J. Clin. Invest. 99, 178-184 (1997)

31. Elsner, J. et al. The CC chemokine antagonist Met-RANTES inhibits eosinophil effector functions through the chemokine receptors CCR1 and CCR3. Eur. J. Immunol. 27, 2892-2898 (1997).

32. Barnes, P. J. \& Lim, S. Inhibitory cytokines in asthma. Mol. Med. Today 4, 452-458 (1998).

33. Selig, W. \& Tocker, J. Effect of interleukin-1 receptor antagonist on antigen-induced pulmonary responses in guinea-pigs. Eur. J. Pharmacol. 213, 331-336 (1992).

34. Pretolani, M. \& Goldman, M. IL-10: a potential therapy for allergic inflammation? Immunol. Today 18, 277-280 (1997).

35. Borish, L. et al. Interleukin-10 regulation in normal subjects and patients with asthma. J. Allergy Clin. Immunol 97, 1288-1296 (1996).

36. John, M. et al. Inhaled corticosteroids increase IL-10 but reduce MIP-1 $\alpha$, GM-CSF and IFN- $\gamma$ release from alveolar macrophages in asthma. Am. J. Respir. Crit. Care Med. 157, 256-262 (1998).

37. van Deventer, S. J., Elson, C. O. \& Fedorak, R. N. Multiple doses of intravenous interleukin 10 in steroid-refractory Crohn's disease. Crohn's Disease Study Group. Gastroenterology 113, 383-389 (1997).

38. Seldon, P. M., Barnes, P. J. \& Giembycz, M. A. Interleukin-10 does not mediate the inhibitory effect of PDE4 inhibitors and other cAMP-elevating drugs on lipopolysacchariude-induced tumor necrosis factor- $\alpha$ generation from human peripheral blood monocytes. Cell Biochem. Biophys. 28 179-201 (1998).

39. Lack, G. et al. Nebulized IFN-gamma inhibits the development of secondary allergic responses in mice. J. Immunol. 157, 1432-1439 (1996).

40. Boguniewicz, M., Martin, R. J., Martin, D., Gibson, U. \& Celniker, A. The effects of nebulized recombinant interferon-y in asthmatic airways. J. Allergy Clin. Immunol 95, 133-135 (1995).

41. Benjaponpitak, S. et al. The kinetics of change in cytokine production by $\mathrm{CD} 4 \mathrm{~T}$ cells during conventional allergen immunotherapy. J. Allergy Clin. Immunol. 103, 468-475 (1999).

42. Durham, S. R. et al. Grass pollen immunotherapy inhibits allergen-induced infiltration of CD4+ T lymphocytes and eosinophils in the nasal mucosa and increases the number of cells expressing messenger RNA for interferon-gamma. J. Allergy Clin. Immunol. 97, 1356-1365 (1996).

43. Gately, M. K. et al. The interleukin-12/interleukin-12-receptor system: role in normal and pathologic immune responses. Annu. Rev. Immunol. 16, 495-521 (1998).

44. Gavett, S. H. et al. Interleukin 12 inhibits antigen-induced airway hyperresponsivness, inflammation and Th2 cytokine expression in mice. J. Exp. Med. 182, 1527-1536 (1995)

45. Kim, T. S. et al. An ovalbumin-IL-12 fusion protein is more effective than ovalbumin plus free recombinant IL-12 in inducing a T helper cell type 1-dominated immune response and inhibiting antigen-specific IgE production. J. Immunol. 158, 4137-4144 (1997).

46. Torphy, T. J. Phosphodiesterase isoenzymes. Am. J. Respir. Crit. Care Med. 157, 351-370 (1998)

47. Chan, S. C. \& Hanifin, J. M. Differential inhibitor effects on phosphodiesterase isoforms in atopic and normal leukocytes. J. Lab Clin. Med. 121, 44-51 (1993).

48. Harbison, P. L. et al. The effect of a novel orally active selective PDE4 isoenzyme inhibitor (CD840) on allergen-induced responses in asthmatic subjects. Eur. Respir. J. 10, 1008-1014 (1997).

49. Muller, T., Engels, P. \& Fozard, J. Subtypes of the type 4 cAMP phosphodiesterase: structure, regulation and selective inhibition. Trends Pharmacol. Sci. 17, 294-298 (1996).

50. Seybold, J. et al. Induction of phosphodiesterases 3B, 4A4, 4D1, 4D2, and 4D3 in Jurkat T- cells and in human peripheral blood T-lymphocytes by 8-bromo-cAMP and $\mathrm{G}_{\mathrm{s}}$-coupled receptor agonists. Potential role in $\beta_{2}$-adrenoreceptor desensitization. J. Biol. Chem. 273, 20575-20588 (1998).

51. Barnes, P. J. \& Karin, M. Nuclear factor-кB: a pivotal transcription factor in chronic inflammatory diseases. N. Engl. J. Med. 336, 1066-1071 (1997).

52. Barnes, P. J. \& Adcock, I. M. Transcription factors and asthma. Eur. Respir. J. 12, 221-234 (1998)

53. Karin, M. Mitogen-activated protein kinase cascades as regulators of stress responses. Ann. N. Y. Acad. Sci. 851, 139-146 (1998).

54. Schafer, P. H., Wadsworth, S. A., Wang, L. \& Siekierka, J. J. p38alpha mitogen-activated protein kinase is activated by CD28-mediated signaling and is required for IL-4 production by human CD4+CD45RO+ T Cells and Th2 effector cells. J. Immunol. 162, 7110-7119 (1999).

55. Kankaanranta, H., Giembycz, M. A., Barnes, P. J. \& Lindsay, D. A. SB203580, an inhibitor of p38 mitogen-activated protein kinase, enhances constitutive apoptosis of cytokine-deprived human eosinophils. J. Pharmacol. Exp. Ther. 290, 621-628 (1999).

56. Costello, P. S. et al. Critical role for the tyrosine kinase Syk in signalling through the high affinity IgE receptor of mast cells. Oncogene 13, 2595-2605 (1996).

57. Yousefi, S., Hoessli, D. C., Blaser, K., Mills, G. B. \& Simon, H. U. Requirement of Lyn and Syk tyrosine kinases for the prevention of apoptosis by cytokines in human eosinophils. J. Exp. Med. 183 1407-1414 (1996).

58. Amoui, M., Draber, P. \& Draberova, L. Src family-selective tyrosine kinase inhibitor, PP1, inhibits both FceRI- and Thy-1-mediated activation of rat basophilic leukemia cells. Eur. J. Immunol. 27, 1881-1886 (1997). 
59. Alexander, A. G., Barnes, N. C. \& Kay, A. B. Trial of cyclosporin in corticosteroid-dependent chronic severe asthma. Lancet 339, 324-328 (1992).

60. Nizankowska, E. et al. Treatment of steroid-dependent bronchial asthma with cyclosporin. Eur. Resp. J. 8, 1091-1099 (1995).

61. Morley, J. Cyclosporin A in asthma therapy: a pharmacological rationale. J. Autoimmunity 5 (Suppl. A), 265-269 (1992).

62. Bieber, T. Topical tacrolimus (FK 506): a new milestone in the management of atopic dermatitis. J. Allergy Clin. Immunol. 102, 555-557 (1998).

63. Thompson, A. G. \& Starzl, T. C. New immunosuppressive drugs: mechanistic insights and potentia therapeutic advances. Immunol. Rev. 136, 71-98 (1993).

64. Pilewski, J. M. \& Albelda, S. M. Cell adhesion molecules in asthma: homing activation and airway remodelling. Am. J. Respir. Cell Mol. Biol. 12, 1-3 (1995).

65. Weg, V. B., Williams, T. J., Lobb, R. R. \& Noorshargh, S. A monoclonal antibody recognizing very late activation antigen-4 inhibits eosinophil accumulation in vivo. J. Exp. Med. 177, 561-566 (1993).

66. Sun, J. et al. Contribution of intracellular adhesion molecule- 1 in allergen-induced airway hyperresponsiveness and inflammation in sensitised Brown-Norway rats. Int. Arch. Allergy Immunol. 104, 291-295 (1994).

67. Yuan, Q., Strauch, K. L., Lobb, R. R. \& Hemler, M. E. Intracellular single-chain antibody inhibits integrin VLA-4 maturation and function. Biochem. J. 318, 591-596 (1996).

68. Lin, K. C. et al. Selective, tight-binding inhibitors of integrin alpha4betal that inhibit allergic airway responses. J. Med. Chem. 42, 920-934 (1999).

69. Abraham, W. M. et al. Selectin blockade prevents antigen-induced late bronchial responses and airway hyperresponsiveness in allergic sheep. Am. J. Respir. Crit. Care Med. 159, 1205-1214 (1999).

70. Kim, M. K., Brandley, B. K., Anderson, M. B. \& Bochner, B. S. Antagonism of selectin-dependent adhesion of human eosinophils and neutrophils by glycomimetics and oligosaccharide compounds. Am. J. Respir. Cell Mol. Biol. 19, 836-841 (1998).

71. Heinke, S., Szucs, G., Norris, A., Droogmans, G. \& Nilius, B. Inhibition of volume-activated chloride currents in endothelial cells by chromones. Br. J. Pharmacol. 115, 1393-1398 (1995).

72. Wang, L., Correia, I., Basu, S. \& Theoharides, T. C. $\mathrm{Ca}^{2+}$ and phorbol ester effect on the mast cell phosphoprotein induced by cromolyn. Eur. J. Pharmacol. 371, 241-249 (1999).

73. Bianco, S. et al. Inhaled loop diuretics as potential new anti-asthmatic drugs. Eur. Resp. J. 6, 130-134 (1993).

74. Yates, D. H. et al. Effect of acute and chronic inhaled furosemide on bronchial hyperresponsivenes in mild asthma. Am. J. Respir. Crit. Care Med. 152, 892-896 (1995).

75. Haczku, A. et al. Anti-CD86 (B7. 2) treatment abolishes allergic airway hyperresponsiveness in mice. Am. J. Respir. Crit. Care Med. 159, 1638-1643 (1999).

76. Van Oosterhout, A. J. et al. Murine CTLA4-IgG treatment inhibits airway eosinophilia and hyperresponsiveness and attenuates $\operatorname{IgE}$ upregulation in a murine model of allergic asthma. Am. J. Respir. Cell Mol. Biol. 17, 386-392 (1997).

77. van Neerven, R. J. et al. Requirement of CD28-CD86 costimulation for allergen-specific T cell proliferation and cytokine expression. Clin. Exp. Allergy 28, 808-816 (1998).

78. Kon, O. M. et al. Randomised dose-ranging placebo-controlled study of chimeric antibody to CD4 (keliximab) in chronic severe asthma. Lancet 352, 1109-1113 (1998).

79. Schwarze, J. et al. CD8 T cells are essential in the development of respiratory syncytial virus-induced lung eosinophilia and airway hyperresponsiveness. J. Immunol. 162, 4207-4211 (1999).

80. Zhang, D. H., Cohn, L., Ray, P., Bottomly, K. \& Ray, A. Transcription factor GATA-3 is differentially expressed in murine Th1 and Th2 cells and controls Th2-specific expression of the interleukin-5 gene. J. Biol. Chem. 272, 21597-21603 (1997)

81. Caramori, G. et al. GATA transcription factor expression in T cells, monocytes and bronchia biopsies of normal and asthmatic subjects. Am. J. Respir. Crit. Care Med. 157, A908 (1999).

82. Murphy, E. et al. Reversibility of T helper 1 and 2 populations is lost after long-term stimulation. J. Exp. Med. 183, 901-913 (1996).

83. Corne, J. et al. The effect of intravenous administration of a chimeric anti-IgE antibody on serum IgE levels in atopic subjects: efficacy, safety, and pharmacokinetics. J. Clin. Invest. 99, 879-887 (1997).

84. Fahy, J. V. et al. The effect of an anti-IgE monoclonal antibody on the early and late phase responses to allergen inhalation in asthmatic subjects. Am. J. Respir. Crit. Care Med. 155, 1828-1834 (1997).

85. Coyle, A. J. et al. Central role of immunoglobulin (Ig) E in the induction of lung eosinophil infiltration and T helper 2 cell cytokine production: inhibition by a non-anaphylactoid anti-IgE antibody. J. Exp. Med. 183, 1303-1310 (1996).

86. Holgate, S. T., Corne, J., Jardieu, P., Fick, R. B. \& Heusser, C. H. Treatment of allergic airways disease with anti-IgE. Allergy 53, 83-88 (1998).

87. Durham, S. R. et al. Long-term clinical efficacy of grass-pollen immunotherapy. N. Engl. J. Med. 341, 468-475 (1999).

88. Hsu, C. H. et al. Immunoprophylaxis of allergen-induced immunoglobulin E synthesis and airway hyperresponsiveness in vivo by genetic immunization. Nature Med. 2, 540-544 (1996).

89. Roy, K., Mao, H. Q., Huang, S. K. \& Leong, K. W. Oral gene delivery with chitosan-DNA nanoparticles generates immunologic protection in a murine model of peanut allergy. Nature Med. 5, 387-391 (1999).

90. Yssel, H., Fasler, S., Lamb, J. \& de Vries, J. E. Induction of non-responsiveness in human allergen specific type 2 helper cells. Curr. Opin. Immunol. 6, 847-852 (1994).

91. Norman, P. S. et al. Treatment of cat allergy with T-cell reactive peptides. Am. J. Respir. Crit. Care Med. 154, 1623-1628 (1996).

92. Haselden, B. M., Barry Kay, A. \& Larche, M. Immunoglobulin E-independent major histocompatibility complex-restricted T cell peptide epitope-induced late asthmatic reactions. J. Exp. Med. 189, 1885-1894 (1999).

93. Shirakawa, T., Enomoto, T., Shimazu, S. \& Hopkin, J. M. The inverse association between tuberculin responses and atopic disorder. Science 275, 77-79 (1997).

94. Strannegard, I. L., Larsson, L. O., Wennergren, G. \& Strannegard, O. Prevalence of allergy in children in relation to prior BCG vaccination and infection with atypical mycobacteria. Allergy 53, 249-254 (1998).

95. Herz, U. et al. BCG infection suppresses allergic sensitization and development of increased airway reactivity in an animal model. J. Allergy Clin. Immunol. 102, 867-874 (1998).

96. Wang, C. C. \& Rook, G. A. Inhibition of an established allergic response to ovalbumin in BALB/c mice by killed Mycobacterium vaccae. Immunology 93, 307-313 (1998).

97. Wheeler, J. G. et al. Immune and clinical impact of Lactobacillus acidophilus on asthma. Ann. Allergy Asthma Immunol. 79, 229-233 (1997).

98. Sur, S. et al. Long term prevention of allergic lung inflammation in a mouse model of asthma by CpG oligodeoxynucleotides. J. Immunol. 162, 6284-6293 (1999).

99. Xing, Z., Ohkawara, Y., Jordana, M., Grahern, F. L. \& Gauldie, J. Transfer of granulocytemacrophage colony-stimulating factor gene to rat induces eosinophilia, monocytosis and fibrotic lesions. J. Clin. Invest. 97, 1102-1110 (1996).

100. Nyce, J. W. \& Metzger, W. J. DNA antisense therapy for asthma in an animal model. Nature 385, $721-725$ (1997) 